43-44 | 2013

Le pastoralisme en Haute-Asie : la raison nomade dans l'étau des modernisations

\title{
Une première approche sur la transmission du chant diphonique en Mongolie
}

A first approach on the transmission of overtone singing in Mongolia

Johanni Curtet

\section{(2) OpenEdition}

Journals

Édition électronique

URL : https://journals.openedition.org/emscat/2123

DOI : 10.4000/emscat.2123

ISSN : 2101-0013

Éditeur

Centre d'Etudes Mongoles \& Sibériennes / École Pratique des Hautes Études

Référence électronique

Johanni Curtet, "Une première approche sur la transmission du chant diphonique en Mongolie », Études mongoles et sibériennes, centrasiatiques et tibétaines [En ligne], 43-44 | 2013, mis en ligne le 20 septembre 2013, consulté le 13 juillet 2021. URL : http://journals.openedition.org/emscat/2123 ; DOI : https://doi.org/10.4000/emscat.2123

Ce document a été généré automatiquement le 13 juillet 2021.

(c) Tous droits réservés 


\title{
Une première approche sur la transmission du chant diphonique en Mongolie
}

\author{
A first approach on the transmission of overtone singing in Mongolia
}

Johanni Curtet

\section{NOTE DE L'AUTEUR}

Je tiens à remercier Hervé Lacombe, Laurent Legrain, Grégory Delaplace, Roberte Hamayon et Isabelle Charleux pour leurs précieux conseils en relisant cet article, Olivier Gassies pour son assistance technique, ainsi que les musiciens avec qui je travaille sur le terrain qui, depuis nos premières rencontres, font que ma recherche existe.

1 Transmis à la campagne comme à la ville par l'oralité de génération en génération, le höömij (chant diphonique) est aussi entré dans l'enseignement universitaire à partir du début des années 1990. Des années 1930 au début du xxI siècle, d'importantes transformations sont survenues dans la pratique de cette technique vocale. Elles sont la preuve de l'existence des mécanismes d'une tradition vivante, persistante et mouvante du passé au présent (Pouillon [1991] 2010, p.710). Me fondant sur des données ethnographiques collectées entre 2004 et 2010, j'aborde la question de la transmission du chant diphonique dans la Mongolie contemporaine. Je m'intéresse aux processus de transmission, mais aussi aux ruptures et changements qui font du höömij une tradition vivace. À travers un regard sur l'histoire et la société mongoles, la limite de mon champ d'investigation est l'observation des modes d'enseignements en milieu rural dans l'Altaï (Altaj) mongol et à l'Université d'Art et de Culture d'Ulaanbaatar.

Une approche ethnomusicologique et historique des évolutions sociales de la musique mongole a déjà été développée par Carole Pegg (2001a), Peter K. Marsh (2009) et Laurent Legrain (2009). Mais aucune étude approfondie sur la transmission du höömij en 
Mongolie n'a vu le jour. À partir d'une leçon de chant diphonique de D. Sunduj (1938-2003), Alain Desjacques étudie le rôle des voyelles de la langue mongole dans l'apprentissage de cette technique vocale $(1988,1991,1992$, pp. 47-117)1. C. Pegg aborde la transmission chez les höömijčid (chanteurs de höömij) halh de Čandman’2 dans la province de Hovd sous l'angle des prédispositions (l'âge, le genre et le physique) nécessaires à l'apprentissage (1992, pp. 42-44). Elle évoque des méthodes d'enseignement employées par les chanteurs de höömij, mais sans en donner le détail, ni en expliquer le rôle. Marc van Tongeren présente succinctement la transmission du chant diphonique touva à travers ses observations de terrain et son expérience d'apprentissage auprès de quelques chanteurs. Il met en avant les principes fondamentaux des enseignements observés : une transmission par imitation dans un cadre familial, mais aussi avec les outils contemporains de diffusion de la musique, comme la radio, la télévision et les enregistrements; le rôle des voyelles dans l'émission des harmoniques, préambule à toute réalisation mélodique; et la constante technique d'une pression exercée sur les cordes vocales et sur certaines parties du corps (1995, pp. 302-304, 308). Enfin, L. Legrain inscrit la question de la transmission de la musique au centre de ses recherches, à partir d'un terrain anthropologique chez les Darhad du Hövsgöl (2010, 2011). Il s'interroge sur les liens particuliers qui unissent les Mongols au chant, et propose l'idée de " continuum sonore». Celui-ci englobe en plus de l'ensemble des pratiques chantées, certains sons de la nature, et les éléments prosodiques de la voix, tous ces éléments étant rassemblés sous le même terme mongol duu (voix). Ainsi, dès le plus jeune âge, l'individu est appelé à être attentif à la " voix ", et apprend ainsi naturellement le chant et ses diverses fonctions (2011, pp. 341-397).

3 Les travaux effectués par les chercheurs autochtones sur le höömij mongol se limitent à l'étude historique et à la description ethnographique (Badraa 1981, 1983, Enebiš 2001, 2002). Signalons la parution en 2009 de plusieurs articles consacrés à divers aspects de la spectacularisation du chant diphonique, écrits par Altangerelyn Ceden-Ǐs et rassemblés dans son ouvrage en deux volumes, Hög/Žim ( La musique) et Hög\#Žil (Le développement). La même année, Valentina Süzükei publie un article sur la transmission du höömij en Touva. Elle présente en contraste deux modes d'apprentissage, formel et informel et montre l'influence de l'institutionnalisation de l'enseignement des traditions (Süzükei 2009)3. En 2010, toutefois, paraissent en Mongolie les deux premiers ouvrages entièrement consacrés au höömij : Mongol höömejn utga holbogdol (L'importance et le sens du höömij mongol) de Lhasürengijn Herlen, et Mongol höömej (Le höömij mongol) d'Erdene-Očiryn Sandagžav. La sortie de ces deux ouvrages se situe sans aucun doute dans la continuité du projet de patrimonialisation de l'État Mongol, qui a inscrit le höömij sur la liste représentative du Patrimoine Culturel Immatériel de l'Humanité à l'Unesco en novembre 2010.

4 Après une présentation succincte de la technique vocale, je mettrai en lumière différents modes de transmission en usage en Mongolie. Je tenterai de montrer quel est le lien entre l'héritage développé dans un milieu rural et sa mutation dans les centres urbains. Il sera possible de distinguer ce qui est conservé et transformé d'une génération à l'autre dans l'enseignement. En parallèle, j'exposerai comment la pratique $\mathrm{du}$ chant diphonique est devenue un enjeu identitaire aux niveaux interethnique, régional, national et international, au sein d'un processus de patrimonialisation déjà en genèse pendant la période communiste, dont les effets ont déjà imprégné le discours de mes interlocuteurs. 


\section{Une définition du chant diphonique mongol}

Avant de montrer quels sont les éléments fondamentaux qui entrent en jeu dans la transmission, il convient d'expliquer le principe de cette technique vocale. En effet, sa pratique et son enseignement doivent être abordées comme un tout qui est transmis et à l'intérieur duquel le höömij prend forme.

On localise le chant diphonique dans plusieurs parties du monde, notamment en Asie centrale, dans les montagnes de l'Altaï et leurs environs. Le höömij se retrouve dans les régions russes du Gorno-Altaï, de Khakassie et de Touva (Léothaud 1989, p. 36, Trân Quang 1995, pp. 125-127, Pegg 1992 p. 32), en Mongolie surtout dans les provinces de l'ouest (Zavhan, Hovd, Uvs et Bajan-Ölgij), au nord du Xinjiang et en MongolieIntérieure en Chine ${ }^{4}$. En Mongolie, plusieurs groupes ethniques, tels que les Halh, Irged (Touva), Altaj Urianhaj, Zahčin, Bajad, Dörvöd et Hotgojd pratiquent le chant diphonique à l'heure actuelle. Le höömij s'est diffusé à l'échelle du pays depuis les années 1990.

7 Höömij ${ }^{5}$ est le terme employé par les Mongols pour décrire la technique vocale d'une seule personne superposant volontairement plusieurs sons simultanément avec sa voix, mais principalement deux sons distincts: une mélodie d'harmoniques réalisée audessus d'un son fondamental, appelé bourdon. Les harmoniques viennent du bourdon vocal et sont extraits grâce à une contraction simultanée du pharynx et du diaphragme (Trân Quang, 1995 p. 123, 125). Pour effectuer une mélodie d'harmoniques, le höömijč (chanteur de höömij) module sa cavité buccale de plusieurs manières, que j'exposerai plus loin.

8 En mongol, höömij signifie littéralement « pharynx » (Legrand et Legrand-Karkucinska 2007, p. 557). C'est l'une des parties principales de l'appareil phonatoire qui agit pour produire cette technique vocale. Autrefois le höömij était réservé exclusivement aux hommes. Un interdit reposait sur la pratique des femmes qui pouvaient en perdre leur fertilité ou parce qu'elles n'avaient pas assez de puissance pour réaliser un chant nécessitant autant de force physique (Pegg 2001a, pp. 108, 172). Avec l'ouverture du pays en 1990 et un changement dans les mentalités déjà apparent pendant les années 1980, quelques femmes ont commencé à apprendre le höömij, à Čandman' d'abord avec Dašdoržijn Cerendavaa (Pegg 1992, p. 43, Desjacques 1992, p. 13), puis à Ulaanbaatar avec Baataryn Odsüren dans les années 1990 et plus récemment à Ulaangom avec Ežeegijn Tojvgoo. Selon ces maîtres et quelques doyens, un bon höömijč se doit d'être fort, honnête, intelligent, respectueux envers les autres, si possible bon cavalier et bon lutteur. La qualité d'une performance de höömij se remarque dans le choix du timbre diphonique, l'évocation possible d'un milieu naturel, la capacité respiratoire ${ }^{6}$, la puissance de la voix et enfin la beauté mélodique. Ces références se retrouvent en général dans l'appréciation du chant sous ses multiples aspects en Mongolie (Legrain 2007, pp. 6-7, 2011, pp. 143, 267, 307, 466-469).

9 Il existe une multitude de techniques de chants diphoniques. Elles se regroupent sous deux styles principaux : le harhiraa (höömij profond ${ }^{7}$ ) et l'isgeree höömij (höömij sifflé). Lors de l'émission harhiraa, le höömijč chante un bourdon avec une voix de gorge puis, en pressant simultanément sur le pharynx et l'abdomen, après avoir pris une bonne inspiration, il réalise un son grave qui vibre une octave inférieure au son fondamental produit. Ce son grave et rauque que l'on entend au premier plan et qui caractérise le 
style harhiraa est dû à la vibration simultanée des cordes vocales et des bandes ventriculaires. Par-dessus cette double-basse, le höömijč réalise une mélodie d'harmoniques aigus. L'émission isgeree höömij est aussi appelée narijn höömij (höömij aigu), ujangyn höömij (höömij mélodieux) ou Altajn šingen höömij (höömij « liquide » de l'Altaï). Le chanteur de höömij émet un bourdon avec une voix de gorge puis, toujours en pressant simultanément sur le pharynx et l'abdomen, après avoir pris une bonne inspiration, réalise un sifflement harmonique mélodique qui peut vibrer plusieurs octaves au-dessus du son fondamental. Dans les deux cas, avec le harhiraa et l'isgeree höömij, la manière de réaliser la mélodie d'harmoniques est commune. On module l'intérieur de la cavité buccale, notamment en ouvrant et en refermant les lèvres, ou en avançant la langue de l'avant vers l'arrière de la bouche en laissant sa pointe collée au palais, ou encore en avançant la partie centrale de la langue d'avant en arrière, la pointe de la langue placée contre la partie inférieure des dents 8 .

10 S'ajoutent à cela des techniques diphoniques pour enrichir le timbre de la voix et d'autres au caractère ornemental. Les höömijčid se plaisent d'ailleurs à les combiner entre elles. Parmi plus d'une vingtaine de techniques existantes, on trouve notamment le höömij de gorge (bagalzuuryn höömij), écho (cuuraj höömij), de nez (hamryn höömij) ou encore, syllabique (dangildah höömij) ${ }^{9}$. Les chanteurs de höömij utilisent l'émission vocale šahalt (pression), appelée aussi šahmal hooloj (voix étranglée) pour chanter les louanges magtaal. Tout en conservant un timbre guttural, cela leur permet de passer aisément de la louange au sifflement harmonique. Pour approfondir cette définition qui ne se veut pas exhaustive, les travaux de Trân Quang Hai (1971, 1989, 1995, etc.) ont largement mis en avant l'étude des techniques et de l'anatomie du höömij ${ }^{10}$.

11 À son origine, l'acte de höömijlöh, «chanter le höömij» ou "diphoner » ${ }^{11}$, n'est pas inscrit dans la forme spectaculaire où on le rencontre le plus souvent aujourd'hui. Plusieurs légendes attribuent la naissance du chant diphonique à l'imitation des sons de l'eau, du sifflement du vent et des chants d'oiseaux. Des musiciens mongols et A. Desjacques (2002) émettent l'hypothèse que le sifflement diphonique aurait pu naître de l'exécution de l'épopée. En effet, dans l'ouest de la Mongolie, chez certaines ethnies, le barde utilise une voix de gorge particulière, appelée argil, proche du timbre que l'on rencontre dans la phase d'installation du chant diphonique. Certains bardes, arrivés en fin de strophe, prolongent leur chant d'un bourdon vocal tenu sur une voyelle, ou en alternant entre plusieurs syllabes. Ce phénomène laisse bien entendre des harmoniques, mais les bardes n'appellent pas cela höömij et leur volonté n'est pas de réaliser une mélodie. Il pourrait alors s'agir d'une forme primitive de chant diphonique.

Exécuté d'abord dans un contexte pastoral, on utilise le chant diphonique pour imiter la nature, passer le temps en gardant les cheptels, animer une veillée sous la yourte en famille, diphoner des berceuses (Pegg 1992, pp. 35-36, 2001a, p.60; Tongeren 1995, pp. 297-298); ou encore, rejoignant mes observations de terrain, lors de certaines cérémonies domestiques, telles que les mariages, la fête d'une victoire au Naadam. Dans les provinces de l'ouest, la présence du höömij dans ces deux derniers contextes n'est pas systématique. Si un höömijč est présent dans l'assemblée, celui-ci montrera son talent pour le plaisir de l'auditoire et de ses hôtes, comme chaque invité se doit habituellement de boire, manger et chanter pendant de tels événements. C. Pegg mentionne aussi l'usage du höömij par les pasteurs touva et par certains Bajad, pour communiquer respectivement avec leurs yaks et leurs chameaux (Pegg 2001a, p. 236). 
Citant Sanžim, elle rapporte que les chasseurs caatan utiliseraient aussi le höömij à la chasse (ibid., pp. 61, 245) ${ }^{12}$.

Le répertoire du chant diphonique mongol est constitué de reprises de mélodies appartenant à d'autres répertoires vocaux, comme les chants courts (bogino duu), les chants longs (urtyn duu) et les chants populaires (ardyn duu). Il s'agit donc d'un répertoire d'emprunts. Sans se référer à des mélodies particulières, certains höömijčid se plaisent à improviser en « imitant la nature ». Mais en improvisant, ils restent dans le cadre des échelles pentatoniques anhémitoniques, caractéristiques des mélodies populaires mongoles. Dans le répertoire des chants de louanges magtaal, on rencontre aussi du höömij, utilisé comme intermède mélodique entre les couplets. En Mongolie, l'introduction du chant diphonique dans les magtaal serait une pratique récente, remontant aux changements apportés par l'orchestration des musiques « traditionnelles » pendant la période soviétique.

\section{Pratiques et transmissions traditionnelles rurales}

\section{La transmission des années 1930-1960}

Les techniques de chants diphoniques et les répertoires sont transmis de bouche à oreille, de génération en génération. Au regard des archives consultées dans les provinces de Hovd, Zavhan et Ulaanbaatar ${ }^{13}$, il ne semble pas exister pour le moment de documentation, enregistrée ou écrite, expliquant comment le höömij était transmis avant les années 1930. Heureusement, les plus âgés de mes informateurs rencontrés sur le terrain se rappellent de la pratique d'autrefois. Grâce à leur mémoire, on peut obtenir une idée du processus de transmission au cours de leur enfance. J'ai donc assemblé les informations obtenues auprès de trois pasteurs halh, Dugerijn Daržaa (1933-), Gombocedengijn Margad (1939-2005) et Mjatavyn Šagž (1939-2010), résidant tous dans les environs de Čandman'. Leurs témoignages sont à écouter avec prudence. L'influence de la politique des années 1960, promouvant la mise en avant des coutumes représentées comme des folklores afin de faire ressortir "l'unicité mongole » (Aubin 1993, p. 141), suivie d'un esprit de patrimonialisation ambiant en Mongolie depuis les dix dernières années, sont à prendre en compte dans l'idée que tout cela ait participé à la construction d'une tradition mongole généralisée. Mais n'ayant pas d'alternative ici pour compléter ces témoignages oraux avec d'autres sources, je dois tout de même considérer les traces conservées par les anciens pour combler mon questionnement.

Ainsi, l'apprentissage du höömij se faisait par l'imitation d'une personne issue d'un entourage proche, dans les moments de réunions collectives autour du travail de la moisson pour préparer l'hiver, des caravanes de chameaux pour aller chercher du riz et de la farine, de la garde des troupeaux et des diverses activités agricoles ou de construction imposées par le régime communiste. Il existait parallèlement une passation pratiquée sous la yourte. Ces modes de transmission informelle étaient et sont toujours appelés "l'école de la maison» (gerijn surguul'), un terme qui montre l'importance de l'imprégnation de la vie quotidienne. La personne modèle pouvait donner quelques remarques directives pour assurer de la bonne voie à suivre. On utilisait un bol ou les mains, que l'on agitait devant la bouche afin de mieux s'entendre en obtenant de l'écho (cuuraj), dans le but d'améliorer sa technique. À cette époque, apprendre le chant diphonique était un acte expérimental. Il n'y avait pas d'exercices 
spécifiques relatifs à une pédagogie élaborée. Chacun devait trouver par lui-même en suivant les indications des aînés : à savoir, comment prendre son inspiration et gérer son souffle, contracter les muscles de sa gorge et placer sa langue dans la cavité buccale pour moduler les harmoniques. En ce temps, les höömijčid n'étaient pas nombreux à Čandman'. On se rappelle des frères T. Derem (1893-1966) et T. Čuluun (1896-1968), de H. Ceren dit Ceren Gogol (1927- ?), puis de S. Cedee (1924-2004).

Photo 1. D. Daržaa montrant l'utilisation du bol comme résonnateur dans l'apprentissage du höömij

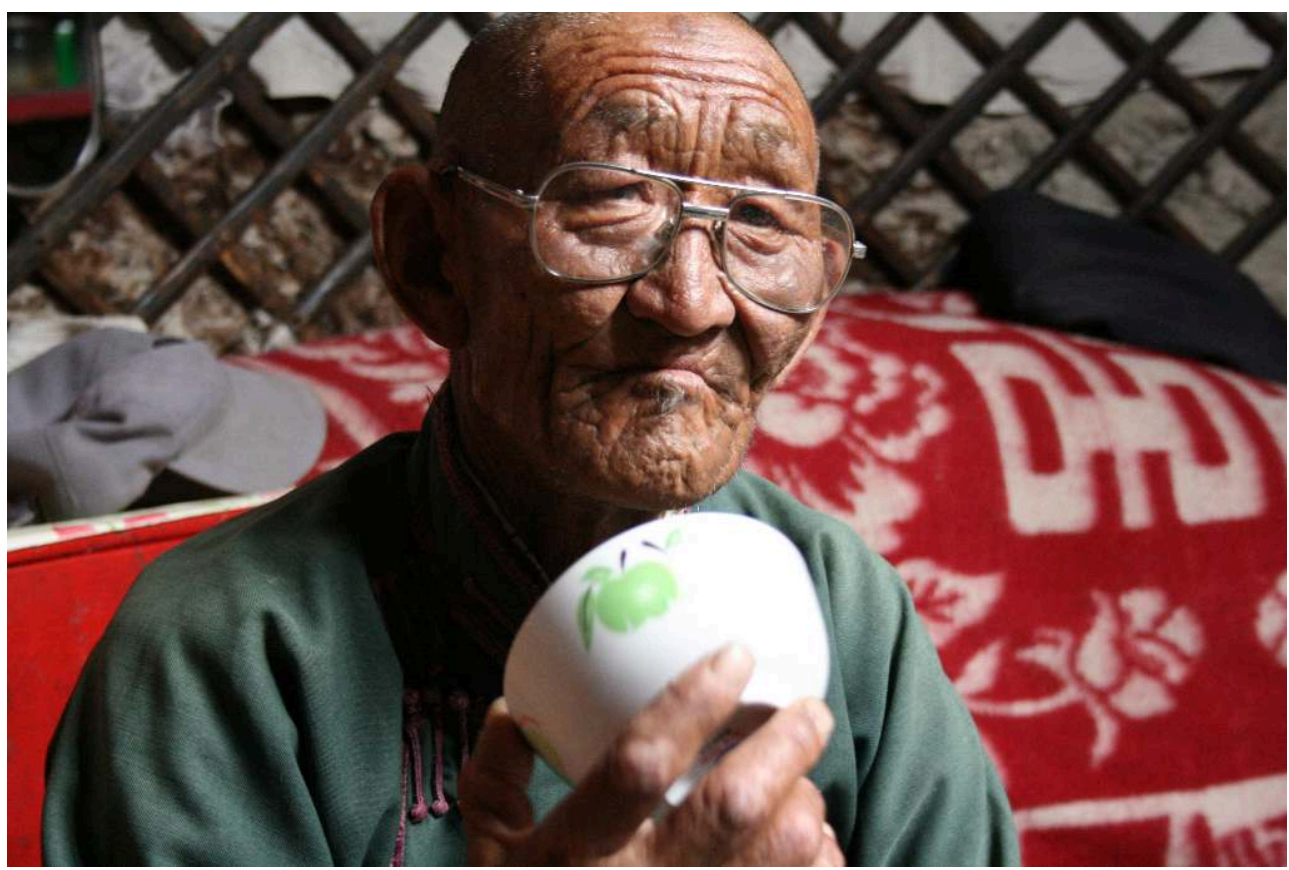

Johanni Curtet (septembre 2010, Čandman', province de Hovd)

Daržaa, Margad et Šagž estiment aussi qu'au moment de leur enfance, la technique vocale et les variantes techniques du chant diphonique n'étaient pas aussi développées qu'aujourd'hui. Selon eux, on ne pratiquait que l'isgeree höömij (chant diphonique sifflé), appelé aussi šahaltin höömij (chant diphonique pressé) et le harhiraa. Ces höömijčid précisent qu'à cette époque, ils avaient l'ambition de diphoner mieux que la génération précédente. Rappelons qu'il s'agit d'une période où, selon l'idéologie socialiste, le peuple était constamment mis en concurrence pour obtenir de lui les meilleurs résultats. Cela s'est vérifié dans les milieux de l'industrie et de l'agriculture avec les coopératives de l'économie collective où chaque ferme se devait d'être toujours plus performante que les autres (Aubin 1967, p. 146) ; mais aussi dans les arts et la musique, avec des concours ${ }^{14}$ organisés par l'État pour élire les meilleurs représentants de la commune, de la province et du pays tout entier, qui étaient érigés comme des héros (Pegg 2001a, pp. 266-280). Cette concurrence entre les chanteurs a incontestablement participé au développement du höömij. Pour être le meilleur aux compétitions et se distinguer des autres, on développe la technique vocale en cherchant à diphoner des mélodies plus complexes ou en créant de nouvelles techniques de chant diphonique. Par la suite, la place que va prendre le monde de la scène dans l'environnement des chanteurs, soumis à la sélection des directeurs des théâtres et des ensembles, va accélérer ce processus. Aujourd'hui, cette rivalité est devenue très apparente, pour des raisons liées aux enjeux économiques du capitalisme instauré à partir de 1990, suite 
logique de l'entrée progressive du höömij dans l'industrie culturelle (tournées de concerts, production de disques). Cette entrée, que nous allons examiner de plus près, a commencé avec des nouveaux lieux et contextes de représentations.

\section{La transition de la steppe à la scène, années 1950-1970}

La génération des höömijčid des années 1930 et la suivante ont connu le développement d'une nouvelle identité et idéologie culturelles avec l'installation du communisme. Rappelons que c'est entre les années 1920 et 1960 que les ulaan bulan (coins rouges), les klub (clubs) et les ulaan ger (yourtes rouges) ont été déployés par le régime dans les zones rurales de Mongolie pour diffuser l'idéologie marxiste-léniniste (Pegg 2001a, p. 253 et Legrain 2009, p. 7), dans le but d'unifier les populations autour de pratiques artistiques politiquement contrôlées, orientées et correctes (Bawden [1968] 1989, pp. 375-377). Les théâtres sont apparus progressivement dans chaque province et se sont largement développés à Ulaanbaatar, avec un fonctionnement fondé sur le modèle russe et occidental ${ }^{15}$.

À cette période, selon mes informateurs, la transmission du chant diphonique ne dépassait pas le cadre de l'imitation d'un entourage familial ou d'un voisinage proche, notamment dans les provinces de l'ouest, à Zavhan, Hovd, Uvs et Bajan-Ölgij. Pour créer une identité nationale, en développant les musiques traditionnelles à l'image de l'idéologie socialiste, la facture instrumentale, les modes de jeux et les textes des chants ont été transfigurés en suivant l'exemple du grand frère russe, avec de nombreuses influences occidentales (accord des instruments sur le diapason classique du La- $440 \mathrm{~Hz}$, position de jeu de la vièle adaptée à celle du violoncelle, apport de l'harmonie et des gammes tempérées dans les compositions, etc.), tout en conservant une identité mongole nationale (maintien de l'échelle pentatonique-anhémitonique traditionnelle, rythmes inspirés des allures des animaux, évocations de la nature dans les textes). Ainsi, ces musiques transmises dans des contextes spécifiques ont été déplacées sur les scènes des théâtres, dans les villes des provinces et notamment à la capitale, dissociées de leurs contextes d'origine, pour construire la musique d'une nation uniforme.

Selon les témoignages recueillis sur le terrain, S. Cedee, qui aurait appris des frères Derem et Čuluun, a été le premier Halh de Čandman' à présenter le höömij sur la scène du théâtre de Hovd en 1954. Le compositeur D. Luvsanšarav en charge du théâtre à l'époque eut l'idée d'intégrer cette technique vocale à un arrangement chorale du "Chant de louanges à l'Altaï » (Altajn magtaal), composé spécialement pour les décades de Hovd (Sandagžav 2010, pp. 40, 120 ; Luvsanšarav 2011, communication personnelle). Par la suite, de nombreux chanteurs de höömij ont repris ce magtaal qui est devenu une pièce incontournable de leur répertoire. Jusque dans les années 1960, la présence d'un chanteur de höömij dans le programme d'un concert était encore rare. Aussi originaire de Čandman', D. Sunduj a succédé à Cedee et G. Čimeddorž (1931-1980) ${ }^{16}$. À partir du début des années 1960, Sunduj devint rapidement un modèle pour les générations suivantes. Progressivement dans les années 1970 et 1980, chanter le höömij est devenu un nouvel enjeu économique pour les pasteurs qui pouvaient être rémunérés en jouant au théâtre, ou en participant à une tournée de concert à l'étranger, sous l'organisation et le contrôle de l'État. C'est à travers cette tendance à la professionnalisation que les pratiques du chant diphonique se sont rapidement transformées. Nés sous le communisme, les musiciens ont transmis leur savoir à travers le prisme de cette 
politique et des nouvelles manières de penser qu'elle a imposées. Ainsi, avec la mise en avant de la pratique de certains individus halh, venus de Čandman' pour la plupart, remarqués dans les concours de chant et devenus les habitués des théâtres, des enseignements plus personnalisés sont progressivement apparus. Grâce à cela, avec le renforcement de la représentation des traditions sous le communisme, repris plus tard par la politique de patrimonialisation, dans les mentalités, Čandman' est devenu dans les mentalités le lieu d'origine incontesté du höömij et le point de départ du renouveau de la pratique (Pegg 1992, pp. 40-42).

Nous allons voir que dans la dynamique d'un tel contexte, des chanteurs de la région de Čandman' et ceux d'autres provinces de l'Altaï ont développé des modes d'enseignement spécifiques et fondé de véritables écoles de tradition orale, reconnues comme telles aujourd'hui par la communauté des höömijčid. Ils ont ainsi participé à l'évolution des techniques, des répertoires et des contextes de jeu. Je vais maintenant montrer comment cette génération a marqué une étape décisive dans l'orientation de la « tradition» que nous connaissons aujourd'hui.

\section{Transmissions du höömij individualisées, années 1980-1990}

\section{À la campagne}

21 Fondés sur le même principe que "l'école de la maison ",en conservant les éléments idiomatiques de la pratique du chant diphonique, des höömijčid ont proposé de nouvelles manières d'enseigner. En élaboration à la fin des années 1970, ils les ont développées entre les années 1980 et 1990. De cet héritage, les jeunes revendiquent actuellement l'apprentissage auprès d'un maître, souvent issu de la même ethnie. Pourtant, lorsque les aînés de leurs professeurs rendent compte de leur propre formation quarante ans plus tôt, ce sentiment d'appartenance à un maître ou à une ethnie en particulier est absent. Bien qu'ils puissent avoir cité des modèles, la pratique était fondée sur l'imitation, sans suivre de cours ni pratiquer des exercices spécifiques régulièrement.

À Čandman', plusieurs pasteurs halh ont progressivement élargi leur enseignement à un grand nombre de personnes. Pour prendre l'exemple le plus significatif dans cette région, D. Cerendavaa (1954-) a appris le höömij de T. Čuluun, Margad, Šagž et Sunduj, à une époque où les exercices n'étaient pas encore en usage. Cerendavaa raconte qu'il lui aura fallu plus d'un an et demi avant de comprendre comment contracter les muscles de sa gorge et encore quelques mois avant de parvenir à un timbre diphonique qui lui semblait convenable. Si Cerendavaa est devenu un important transmetteur du höömij aujourd'hui, c'est parce qu'il ne voulait pas que la génération suivante se retrouve face aux mêmes difficultées. Dans sa jeunesse, il pensait déjà à transmettre différemment et souhaitait que tout le monde puisse apprendre plus facilement.

Ainsi, depuis la fin des années 1970, il dit avoir transmis le höömij à environ 200 élèves ${ }^{17}$. Il a élargi l'apprentissage aux femmes et aux étrangers. L'ouverture du pays au tourisme en 1990 a contribué à développer l'enseignement du höömij aux voyageurs de passage, qui ont pu connaître cette musique grâce aux tournées et aux disques réalisés en Europe, aux États-Unis et au Japon notamment à partir de la fin des années $1960^{18}$. 

des exercices pour faire progresser l'élève dans des directions précises. On commence par apprendre à contracter la gorge afin de trouver le timbre idéal pour diphoner. On doit rechercher une hauteur de bourdon correspondante à sa tessiture. Cerendavaa insiste particulièrement sur ces deux aspects, avant de passer à l'étape suivante. L'élève doit prononcer en boucle les sept voyelles de l'alphabet mongol (A-E-I-O-U-Ö-Ü) avec un timbre de gorge, en mettant les lèvres en avant, afin de favoriser la sortie des harmoniques. Ensuite, on commence à délier et muscler la langue avec un exercice de prononciation toujours basé sur la succession des voyelles, en y ajoutant les sons « $\mathrm{L}$ » et « $G$ ». Sur un bourdon guttural, on chante le temps d'un souffle l'exercice suivant : langele, lengele, lingele, longele, lungele, löngele, lüngele. Tout en travaillant son timbre guttural (bourdon et prononciation du son « $G »$ ), cela permet d'exercer sa langue contre le palais (lorsque l'on prononce les « $\mathrm{L} »)$. En prononçant la lettre « $\mathrm{L} »$, on place naturellement la pointe de la langue contre la partie osseuse du palais. Une fois la langue disposée ainsi, on doit contracter le pharynx et le larynx davantage pour faire sortir les harmoniques. Tant que ces exercices ne sont pas correctement acquis, Cerendavaa n'enseigne pas de mélodie. Une fois que l'élève commence à diphoner des mélodies, il le fait travailler ensuite sur différentes techniques de chant diphonique. Les notions d'ornementation mélodique viennent plus tard, lorsqu'un certain nombre de techniques est acquis. Parallèlement à cette progression, il conseille aux élèves de diphoner face au vent ou d'imiter le son de la cascade, du torrent ou du ruisseau. Ainsi, comme l'évoquent les légendes, en se mesurant à la nature, le corps apprend intuitivement à trouver la puissance de voix et le timbre recherchés dans le chant diphonique.

Comme tous les höömijčid de sa génération, Cerendavaa connaît et classifie plusieurs sortes de höömij, sans vraiment établir de distinction entre un style et une technique. Il pratique sept « sortes » (töröl) de höömij ${ }^{19}$ : de gorge (bagalzuuryn höömij), palatal (tagnajn höömij), de nez (hamryn höömij), de lèvres (uruulyn höömij), de poitrine (cežnij höömij), profond (hargia höömij) ${ }^{20}$ et combiné (hosmolžin höömij). Il ne transmet pas tout son savoir aux élèves, à l'exception de deux de ses fils, Cogtgerel (1990-) et Haša-Erdene (1996-).

26 Pour construire son enseignement, il dit avoir gardé le meilleur de chacun de ses modèles (un timbre, une technique, des manières de placer la langue dans la bouche pour moduler) et reconstitué le tout à travers des séries d'exercices, en distinguant plusieurs techniques de chant diphonique.

Au sein de sa génération, Cerendavaa fait partie des rares pasteurs à avoir conservé un mode de vie nomade et poursuivit une activité ponctuelle dans l'industrie culturelle. En l'espace de quarante années, Cerendavaa a participé à l'enregistrement de sept disques ${ }^{21}$, est apparu dans plusieurs films documentaires ${ }^{22}$ et a donné quelques dizaines de concerts dans le monde entier, notamment aux États-Unis, au Japon, en Russie et en Europe. 
Photo 2. Erdenečimeg s'exerce au höömij devant sa yourte, en suivant les conseils de D. Cerendavaa

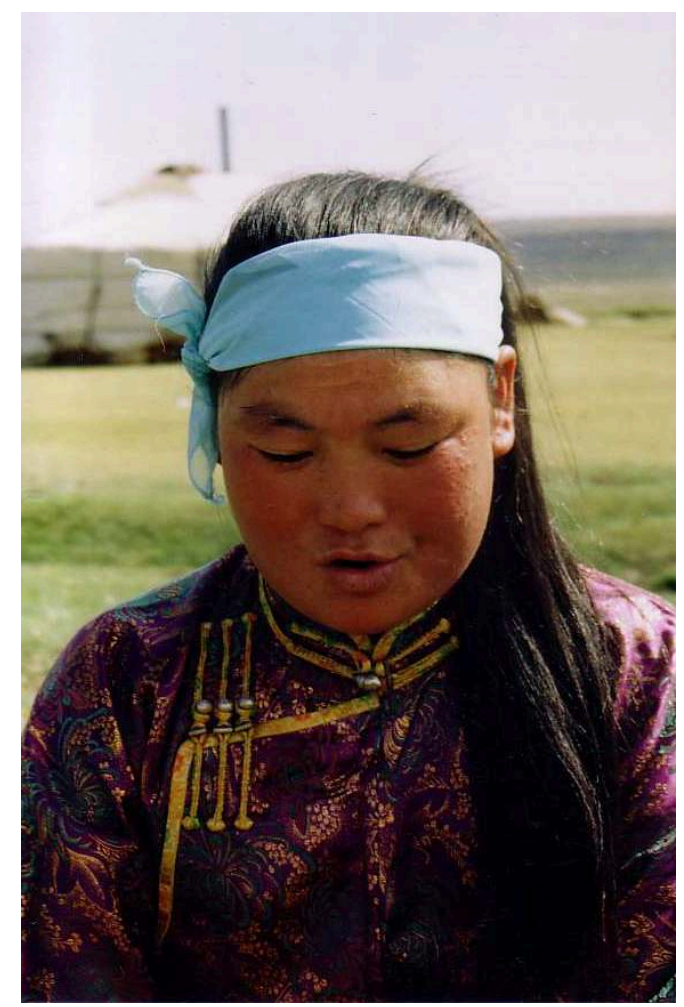

Johanni Curtet (été 2004, Čandman', province de Hovd)

\section{Dans les centres urbanisés en milieu rural}

Le district de Čandman' a tenté de centraliser la pratique du höömij et de l'institutionnaliser pour renforcer la couleur locale et l'attrait touristique. Pour ce faire, un nouveau centre culturel, destiné en partie à l'enseignement du höömij, a été ouvert à l'automne 2009. Malgré cela, depuis la fin des années 1970, de nombreux höömijčid, enfants de pasteurs ayant appris à la campagne, ont saisi l'opportunité de vivre de leur musique et se sont installés à Hovd, la ville de la province, ou à la capitale lorsque l'occasion d'entrer dans un théatre ou un ensemble s'est présentée. À l'exception de Cerendavaa, c'est le cas de la plupart des grands höömijčid actuels, comme les Halh Taravžavyn Ganbold (1957-2011), installé à Ulaanbaatar et Nanžidyn Sengedorž (1948-), établit à Hovd; le Bajad Ežeegijn Tojvgoo (1957-), originaire du district de Zuungov' et résidant à Ulaangom dans la province de l'Uvs; ou encore le Touva irged Badaryn Bapizan (1957-), sédentarisé au village de Cengel à Bajan-Ölgij.

Sengedorž et Tojvgoo ont tous deux étudié à la campagne, respectivement dans les années 1950 et 1960. Dans sa jeunesse, en apprenant le métier de comédien, Sengedorž est devenu aussi höömijč au théâtre de Hovd et représentait le höömij des Halh. Avec un parcours similaire, Tojvgoo incarnait celui des Bajad au théâtre d'Ulaangom. Ils ont commencé à transmettre le chant diphonique à partir de la fin des années 1980 . Sous l'influence du fonctionnement des théâtres, dans lesquels ils apprenaient des exercices d'échauffement pour la chorale, et avec la conception des spectacles fondés sur le modèle soviétique, ils ont développé des modes d'enseignements particuliers, avec de 
nouveaux exercices. Sengedorž, par exemple, a apporté un exercice de prononciation pour muscler la mâchoire, les lèvres et la langue, directement calqué sur les exercices de diction en usage dans l'apprentissage des acteurs. En conservant l'idée d'une succession de voyelles, il a combiné celles-ci avec des consonnes de l'alphabet mongol: a sa pa da ra ma ša ; e se pe de re me še, i si pi di ri mi ši ; o so po do ro mo šo; etc. L'exercice peut se chanter sur un bourdon continu avec l'émission vocale pressée šahalt ou l'émission diphonique harhiraa.

30 Au théâtre d'Ulaangom, Tojvgoo enseigne autour d'un piano. L'exercice d'enchaînement des voyelles se fait en montant et en descendant par ton ou par demiton sur le clavier tempéré. Il fait travailler les chanteurs sur leur tessiture harmonique. Réputé pour être le höömijč au souffle le plus long de Mongolie, il a développé des exercices en soufflant sur l'eau d'un bol ou sur la flamme d'une bougie à différentes distances, afin de contrôler la gestion du mouvement respiratoire.

31 L'ouest de la Mongolie est réputé pour la diversité de ses formes artistiques, réparties chez plusieurs groupes ethniques. Jusqu'ici, il semblerait que la pratique du chant diphonique ait été restreinte aux Halh et à quelques Bajad. Pourtant, selon les recherches de C. Pegg $(1992,2001 a)$ et mes données de terrain, une tradition de chant diphonique qui remonterait avant les années 1920 persiste chez les Zahčin et les Touva. Il s'avère impossible de connaître davantage la forme que prenaient ces pratiques de höömij au pré-communisme. Les purges culturelles et les interdits contre la religion menés par la politique du Parti (Bawden [1968] 1989, pp.350-351, 359-373 et Aubin 1993, p. 143), accompagnés d'une tendance à la domination halh pendant la période communiste (Aubin 1993, p. 159 et Bulag, 1998, pp. 70-80) masquent le passé. Bien que des traces subsistent encore de nos jours, l'existence du höömij chez ces ethnies reste peu considérée par les Halh.

Prenons le cas des Zahčin de la province de Hovd. Le barde T. Enhbalsan (1940-2008) pratiquait le höömij, transmis par son père Tümen-ölzij. Le frère d'Enhbalsan, T. Erdenbüren (1951-) relate que, pendant leur enfance, le höömijč Balžinnjam aurait encouragé leur génération à diphoner. Aussi, à la même époque, deux bardes, Širendev et Vangir, utilisaient le höömij dans leurs épopées ${ }^{23}$. Le manque de sources ne permet pas d'en savoir davantage sur les spécificités de la pratique zahčin. En effet, aujourd'hui, les Zahčin résidant dans la ville de Hovd ou dans les districts de Manhan et de Möst apprennent le chant diphonique en cours particuliers ou dans des stages organisés par les centres culturels avec des höömijčid halh, comme Sengedorž et Cerendavaa, qui sont régulièrement invités. Les jeunes ne se réfèrent donc plus à une lignée Zahčin si tant est qu'elle ait existée. Continuons avec l'exemple des Touva. La rivalité touva/mongole quant à la paternité du chant diphonique ne date pas d'aujourd'hui ${ }^{24}$. Ainsi, une minorité touva résidant en Mongolie à Cengel dans la province de Bajan-Ölgij depuis plus de trois cents ans reste pratiquement ignorée au niveau national. Autrefois, Cengel était majoritairement touva, mais ce district s'est progressivement peuplé de Kazakhs, qui formaient $75,6 \%$ de sa population en 2010 face à $30,3 \%$ de Touva ${ }^{25}$. Pour les habitants, le höömij est transmis dans cette région depuis que les Touva y résident. Il est difficile d'en savoir davantage quand on sait que les Touva de Mongolie n'ont jamais été considérés par les Halh pendant la période communiste bien que, à partir de 1979, ces derniers ont nationalisé le pays en niant la diversité ethnique dans les recensements de population (Aubin 1993, p. 159). Bapizan raconte que parmi les nombreux interdits imposés par le régime, la pratique du höömij 
avait été censurée ${ }^{26}$. Ainsi, au début des années 1990, le chant diphonique touva dans cette province était peu diffusé et en déclin. Depuis quelques années seulement, grâce à l'aide de la chercheuse allemande Amelie Schenk, Bapizan enseigne cette technique vocale à une vingtaine d'enfants, avec des cours hebdomadaires à l'école de Cengel. Bien qu'il soit reconnu au niveau régional, on parle encore peu de Bapizan à Ulaanbaatar, car c'est un chanteur touva.

Photo 3. B. Bapizan enseignant le höömij dans sa salle de classe

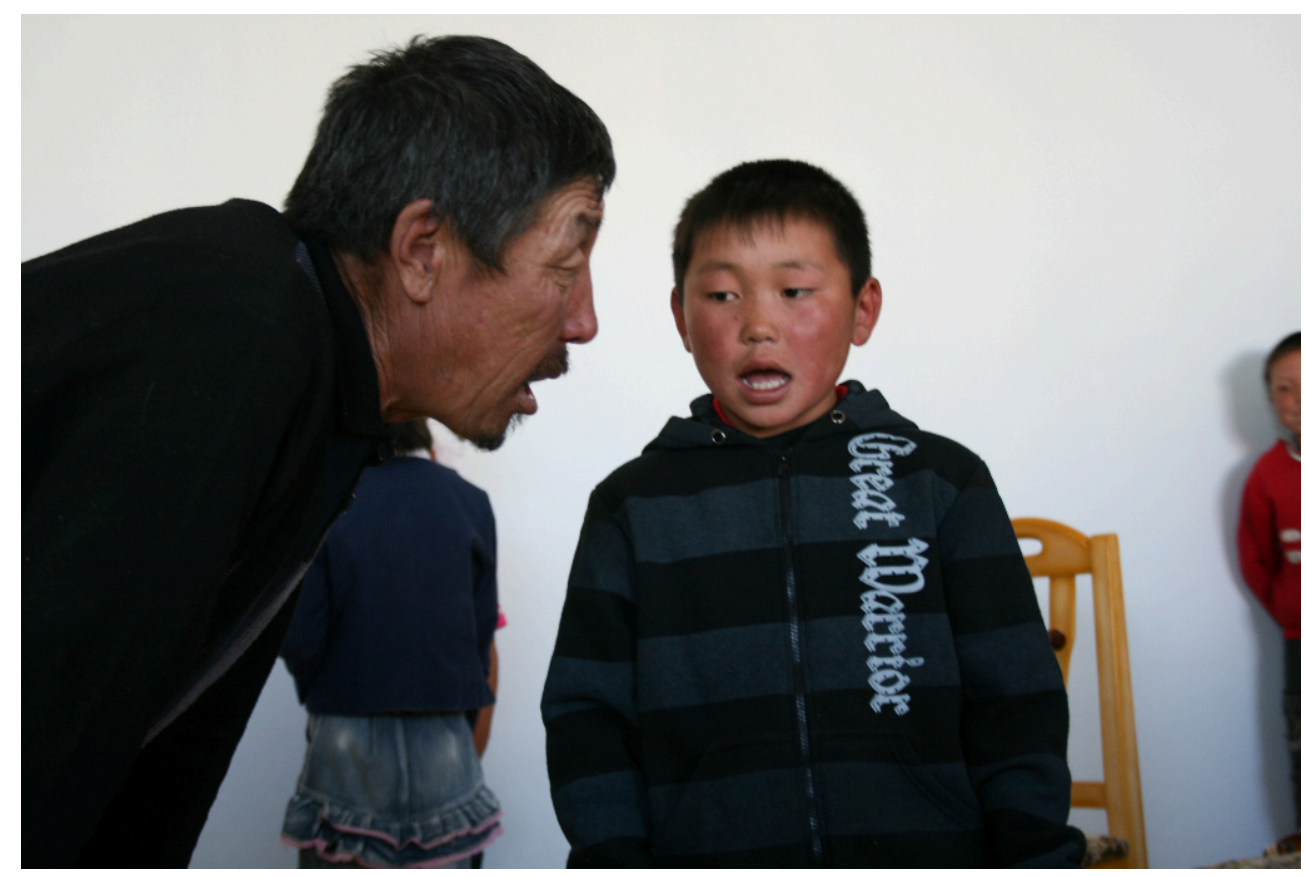

Johanni Curtet (septembre 2010, Cengel, province de Bajan-Ölgij)

La présence du chant diphonique chez ces ethnies montre que nous avons affaire à une tradition de höömij multifacette. Les éléments idiomatiques dans sa transmission, comme le lien à la nature, la gestion du souffle, la contraction recherchée sur la gorge, l'utilisation d'un bol ou de ses mains comme amplificateur pour mieux s'entendre, l'apprentissage de mélodies d'emprunts et certaines valeurs nécessaires pour être perçu comme un bon höömijč, se retrouvent chez les Halh, les Bajad, les Touva et les Zahčin. Mais chacun détient aussi sa spécificité, ce qui fait toute la richesse de la tradition höömij et montre que la pratique a été adaptée en fonction des environnements et des réceptions personnelles de cet héritage. On reste dans un espace commun, les montagnes de l'Altaï, dans une continuité historique commune, celle de la Mongolie, avec des histoires locales et des constructions identitaires multiples. Dès lors que les musiciens sont à Ulaanbaatar, ils représentent une tradition mongole généralisée et mélangée. Ils ne mettront pas en avant au théâtre le fait d'appartenir à une ethnie particulière.

À l'étranger de même, les höömijčid représentent la «nation mongole». Depuis la fin des années 1980, l'État a reconnu l'intérêt du höömij sur la scène internationale. Comme la vièle cheval (morin huur), cette technique vocale est devenue un véritable emblème musical représentant une nation toute entière. Cette tradition multiplie encore ses chemins et devient ici la représentation identitaire d'une nation. Du local au global, le höömij rassemble des spécificités individuelles, familiales et ethniques et devient 
mongol dans sa représentation nationale et internationale. Il appartient désormais aux institutions politiques et culturelles de la Mongolie et plus généralement aux Mongols, dont la construction du concept identitaire « d'être Mongol » remonterait au début des années 1990 (Kaplonski 2004, p. 7). De fait, comme la plupart des instruments et techniques vocales emblématiques, longtemps après l'institutionnalisation de l'enseignement de la vièle cheval, le höömij est la dernière pratique vocale issue du pastoralisme à être entrée dans l'enseignement de "conservatoire ». Nous allons voir quel a été l'apport d'une institution spécifique sur la pratique rurale, inscrivant celle-ci au rang académique et national.

\section{La transmission du höömij institutionnalisée, des années 1990 à nos jours}

\section{À l'université}

Dans la continuité d'un processus d'institutionnalisation des traditions commencé à la période communiste (Marsh 2009, pp. 47-72), c'est en 1992, à l'initiative du musicologue Žamcyn Badraa (1926-1993) ${ }^{27}$, qu'une première classe de höömij est créée à l'Université Nationale de Mongolie. En 2005, une seconde classe délivrant un diplôme de höömijč professionnel ouvre à l'Université d'Art et de Culture d'Ulaanbaatar. Dans chaque université, l'enseignement est confié à B. Odsüren (1949-). Höömijč halh de la province de Zavhan, il est le seul enseignant à transmettre le höömij dans ce contexte en Mongolie ${ }^{28}$.

À l'Université d'Art et de Culture, la transmission se focalise sur une pratique destinée principalement à former des musiciens de scène. Cela influence considérablement les exigences techniques de l'enseignement académique, qui se fonde en général d'abord sur la technicité et la virtuosité, plutôt que de laisser place à l'intuition et la spontanéité, comme L. Legrain le montre à travers l'exemple de l'éducation de l'attention à la voix en milieu rural (2010, p. 62, 2011, pp. 313-416).

Ce cursus est fréquenté par des jeunes étudiants et étudiantes venus de tout le pays. Curieusement, ceux qui ont déjà commencé à apprendre le höömij à la campagne, dans les provinces de l'Altaï, sont les moins nombreux. Des jeunes de Mongolie-Intérieure viennent pour quelques mois ou pour suivre l'ensemble de la formation, ainsi que d'autres étrangers venant du Japon ou de France ${ }^{29}$.

Pendant cette formation de quatre ans, les cours hebdomadaires sont répartis sur quatre niveaux progressifs, sanctionnés par des examens en fin d'année. Odsüren centre sa pédagogie sur des éléments issus de la tradition rurale (la gestion de la respiration et la contraction des muscles de la gorge notamment) qu'il a poussés à l'extrême pour développer la virtuosité des apprentis höömijčid. Aux exercices de prononciation des voyelles, qu'il conserve aussi dans ses cours, il préfère les virelangues, qui permettent aux élèves de muscler à la fois les lèvres, la langue et de travailler la tenue de leur souffle. Leur utilisation, empruntée aux exercices pratiqués dans l'enseignement des épopées (tuul'), est une nouveauté dans l'enseignement du höömij. Odsüren tient cela de son apprentissage personnel des louanges (magtaal) et des récits épiques, dans lesquelles les virelangues sont utilisés pour apprendre à prononcer correctement le texte. Odsüren propose ainsi de nombreuses combinaisons de 
phonèmes à travers ces exercices de prononciation rapide. En voici une série d'exemples:

- Avec le style harhiraa, chanter en comptant en mongol à partir du mot hul (grand bol pour boire l'ajrag) le plus longtemps possible, le temps d'un souffle : $1 \mathrm{hul}, 2 \mathrm{hul}, 3 \mathrm{hul}, 4 \mathrm{hul}, 5 \mathrm{hul}, 6$ hul, 7 hul, 8 hul, 9 hul, 10 hul, etc.

- Même exercice avec un virelangue, à répéter en cycle, en articulant le mieux possible : Minij nutag šüleg, honhor gütger gazar (poème " Mon pays natal », terre de monts et vallées).

- Avec une voix gutturale puissante, prononcer langele, lingele, longele, cycliquement dans un même souffle.

- Imiter le bruit d'une moto ${ }^{30}$. La pointe de la langue est roulée contre les dents de la mâchoire inférieure. Simultanément, la partie centrale de la langue touche la partie osseuse du palais. En maintenant la langue dans cette position, presser la gorge fortement et pousser énergiquement sur le diaphragme en prononçant l'onomatopée trrrrrr.

- Avec le harhiraa ou une voix de gorge contractée, prononcer en alternance le plus vite possible et le temps d'un souffle les mots suivants : dorgoro, dergere, puis : dolgor, delger.

Ces exemples sont l'échantillon d'une véritable pédagogie. De nombreux exercices de gammes avec ou sans diphonie, accompagnés d'un piano, permettent aux élèves d'étendre leur tessiture, comme dans l'apprentissage du chant lyrique. Odsüren suit ses élèves méticuleusement. Il n'hésite pas à adapter ses exercices pour des cas particuliers où les élèves devraient développer un aspect de la pratique qui leur causerait des difficultés. Dans les cours, quel que soit le niveau, on retrouve systématiquement des exercices pour apprendre à gérer son souffle, contracter les muscles de sa gorge, obtenir un timbre guttural, muscler les lèvres et la langue, et progresser à travers l'apprentissage du répertoire. Pour travailler les multiples aspects de la modulation harmonique et faire comprendre les différences de timbre entre les techniques, le maître n'hésite pas à faire passer les élèves d'une technique de chant diphonique à une autre dans un même exercice. Le bon élève devra être capable de diphoner sur des mélodies aux difficultés variées avec plusieurs techniques de höömij. À partir d'une mélodie donnée, telle que Dörvön uul (Quatre montagnes), ou Dörvön nastaj haliun (Le cheval isabelle âgé de quatre ans), le jeune höömijč doit, par exemple, pouvoir la répéter en passant successivement du höömij de nez (hamryn höömij) au höömij écho (cuuraj höömij) et finir en höömij sifflé (isgeree höömij). Plus les techniques diphoniques seront variées plus le höömijč sera impressionnant face à son auditoire. Ce trait est caractéristique de l'évolution d'un monde de l'art professionnel ${ }^{31}$. Mais avant de maîtriser cela, l'apprentissage à l'université est long et exigeant.

En première année, au premier semestre, les élèves travaillent sur la respiration et la contraction de la gorge. Au second semestre, c'est la modulation des voyelles et l'intégration d'une dizaine de mélodies simples issues du répertoire des chants populaires ou des chants courts. Le cours s'intensifie en deuxième année avec un travail autour de mélodies plus complexes. C'est donc à travers l'apprentissage du répertoire que le professeur fait progresser les élèves sur les questions de la contraction de la gorge, de la gestion du souffle et des placements de langue dans les différentes techniques de modulation harmonique. En troisième année, on aborde le demi-ton dans la modulation harmonique. Les élèves doivent être capables de diphoner à la fois dans le pentatonisme anhémitonique de la musique traditionnelle mongole, mais aussi sur des échelles européennes comprenant des demi-tons. Les placements de langue pour atteindre ces derniers sont difficiles. C'est une prouesse technique. En quatrième 
année, les élèves apprennent à diphoner les mélodies des chants longs, et des mélodies étrangères, comme celles de l'opéra italien. Si l'aspect technique semble tenir une grande place dans l'apprentissage, une fois la modulation harmonique maîtrisée, on se concentre aussi sur l'esthétique de la mélodie, en travaillant le vibrato et des ornements harmoniques comme le trille.

Bien qu'il enseigne généralement en classe, Odsüren emmène régulièrement ses élèves dans la nature. À la manière des höömijčid d'autrefois, qui dit-on, pouvaient-être entendus à plusieurs kilomètres alentours en remplissant l'espace de leur chant, les élèves de l'université doivent aussi s'y confronter. Odsüren leur demande de travailler face au vent ou au sommet d'une colline. Cet exercice doit permettre de mesurer jusqu'à quelle distance et avec quelle puissance il est possible de diphoner. En se mesurant à la nature, l'élève doit trouver intuitivement et physiquement une manière de chanter pour s'y égaler. Odsüren passe aussi du temps à expliquer ce que sont les valeurs du höömijč mongol, exposées plus haut. Contrairement à l'enseignement de Cerendavaa qui place la nature et les critères d'appréciation du höömijč au cœur de sa transmission, Odsüren en fait le prétexte d'un cours ou d'une occasion particulière pour une sortie hors de la ville. Les fondements ruraux de la tradition ${ }^{32}$ passent ici après la technique.

Photo 4. Assit devant son piano, B. Odsüren fait travailler ses élèves devant une glace

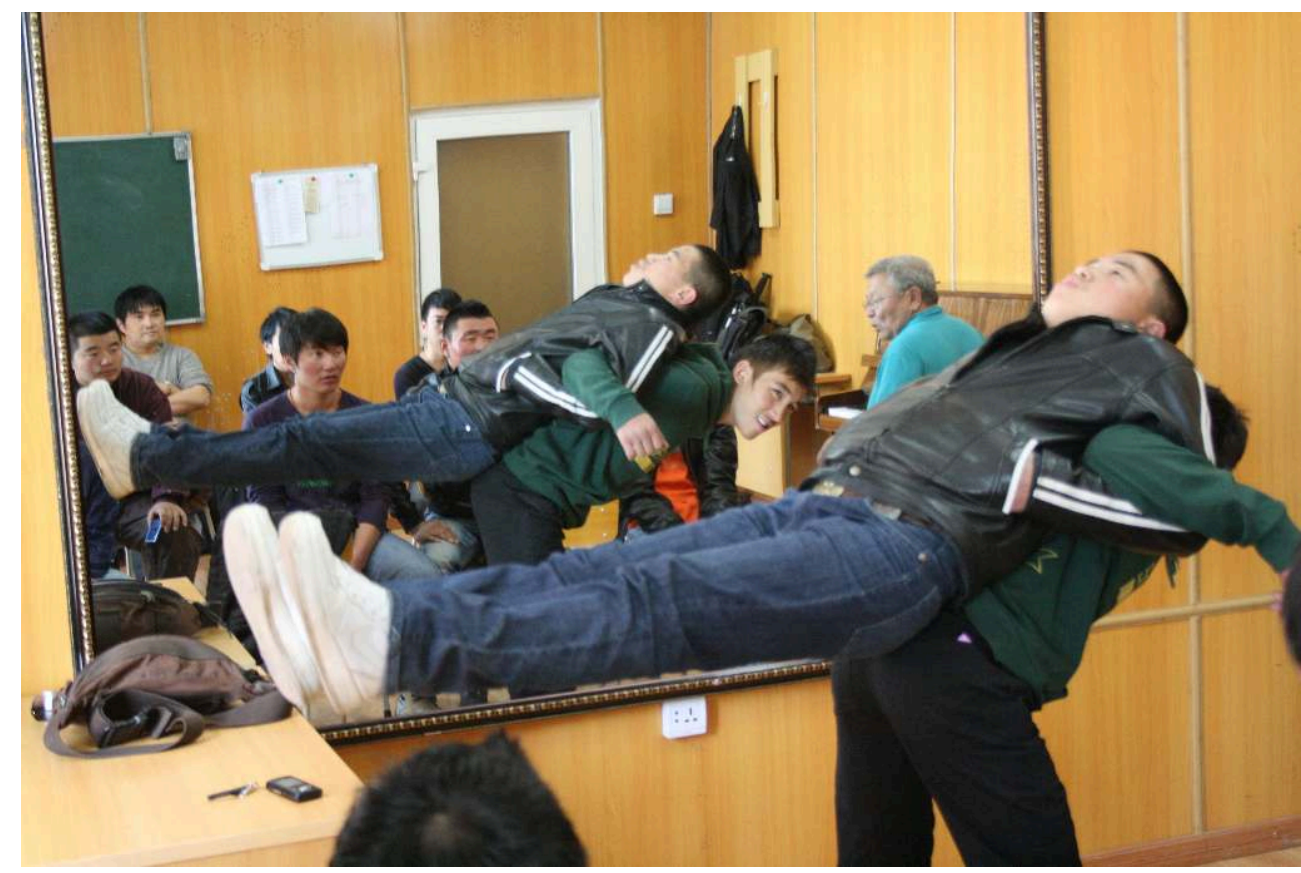

Dans cette position impressionnante, l'élève soulevé par son camarade doit diphoner et utiliser son abdomen correctement pour obtenir un höömij puissant.

Johanni Curtet (septembre 2010, Université d'Art et de Culture, Ulaanbaatar)

En dehors de l'aspect économique qu'apporte cette situation, pour lui, ses élèves et quelques autres chanteurs, être "höömijč professionnel» signifie avant tout être capable de lire une partition en diphonant et de connaître la théorie de la musique, contrairement aux höömijčid ayant appris à la campagne. Les jeunes se démarquent ainsi de la génération qui les précède. Pourtant, certains chanteurs de höömij se considèrent aussi comme " professionnels ", car ils gagnent leur vie avec le höömij. Mais 
on leur reproche constamment de ne pas avoir de diplôme dans leur spécialité. Ainsi, ils ne peuvent pas obtenir officiellement la place d'enseignant ou de höömijč attitré qu'ils souhaiteraient avoir. Le district de Čandman' par exemple, cherche un professeur de höömij pour son nouveau centre culturel. Cerendavaa est le plus compétent dans ce domaine, mais n'a pas de diplôme. La mairie contourne cette difficulté dans ses rapports avec le Ministère, en expliquant que Cerendavaa est employé comme acteur et danseur. Ainsi, il est impossible de reconnaître administrativement que Cerendavaa, l'un des plus grands chanteurs de höömij en Mongolie, enseigne son art chez lui. Pour la même raison, Bapizan ne peut être employé par le centre culturel de Cengel. La directrice du centre préfère employer un jeune touva du village, récemment diplômé du collège de musique de Kyzyl. V. Süzükei remarque le même phénomène à Touva, où cette situation pose un problème aux musiciens traditionnels «non professionnels » qui, dépourvus de statut officiel reconnaissant leur spécialité artistique, sont considérés comme des «amateurs" par les institutions (Süzükei 2009, p.9). En Mongolie, quel que soit l'instrument ou la technique vocale, les musiciens font désormais la distinction entre la transmission "traditionnelle " (ulamžlalt) en milieu rural, et l'apprentissage « professionnel » (mergežlijn).

De nos jours, devenir höömijč peut donner lieu à une reconnaissance nationale, voire internationale. Celui qui apprend mieux que les autres n'est plus perçu comme un pasteur capable de diphoner, mais directement comme höömijč, qualifié le plus souvent de professionnel. La langue mongole fait d'ailleurs la distinction entre un « chanteur de höömij professionnel » (mergežilijn höömijč) et un «talent populaire » (ardyn av'jastan), cette catégorie équivalant à un statut d'amateur. Bien souvent sur mon terrain, en retraçant les liens de maître à élève, j'ai remarqué que les « talents populaires » ont été un jour les modèles des professionnels en exercice aujourd'hui. L'enseignement des musiques mongoles de tradition orale dans les institutions et la fixation des répertoires par l'écriture n'ont fait qu'agrandir l'écart entre les générations. Pour le höömij, cette différenciation est arrivée à son apogée avec l'ouverture successive de ces deux classes de chant diphonique à l'université. Les pasteurs höömijčid le disent : ne sachant pas lire le solfège, ils ne se considèrent pas comme de bons höömijčid, au regard de la jeune génération tournée vers la technicité et la virtuosité. Pourtant, ils connaissent bien d'autres choses. L'imprégnation des espaces naturels qu'ils portent en eux a certainement plus de force dans leur chant que de savoir diphoner le fameux $O$ sole mio d'Eduardo Di Capua, ou un extrait de Carmen de Georges Bizet. Pour la jeune génération, les modèles sont multiples. On respecte les détenteurs de la tradition de l'ouest, mais pour se démarquer, on préfèrera se tourner vers le modèle des prouesses lyriques d'un Pavarotti. Pour d'autres, on ira chercher de nouvelles influences vers le courant ethnorock à la mode à Ulaanbaatar. L. Legrain montre que généralement dans le contexte domestique les critères esthétiques du chant en général sont davantage liés à des émotions de nostalgie pour le pays natal (2011, pp. 86, 458). Ici, l'appréciation du höömij n'est plus la même lorsque l'on passe de la campagne à la ville, bien que cette dernière ait désormais tendance à donner le ton et le rythme à suivre pour l'ensemble du pays.

Une grande partie des nouveaux höömijčid en exercice à Ulaanbaatar est issue de l'université ou des écoles de musique. Mais il existe d'autres cours de höömij dans la capitale, toujours en présence d'un piano, dispensés dans des centres privées, comme Höh tolbo (Tâche bleue mongolique) fondé par T.Ganbold. Jusqu'à aujourd'hui, Odsüren aura été le seul à occuper ce poste à l'université. En mai 2011, il a choisi l'un de 
ces anciens élèves, Zagd-Očir (1981-), pour le seconder. Depuis que le professeur a laissé entrevoir l'horizon d'un départ en retraite, il y a de plus en plus de rivalité entre les élèves de la première promotion de höömijčid professionnels, diplômés en juin 2010, et d'autres plus expérimentés, qui convoitent aussi le futur poste à pourvoir.

\section{En conclusion, la patrimonialisation?}

Une idée constante qui se retrouve chez de nombreux höömijčid est de prendre le meilleur de son modèle, rechercher son propre style et son timbre vocal idéal, puis transmettre à son tour. Le chant diphonique évolue ainsi en permanence, d'un individu à l'autre. Il y aura toujours quelqu'un pour apporter une innovation, une nouvelle manière de faire résonner les harmoniques, et peut-être inventer une nouvelle technique. Tant que le höömij sera pratiqué et perpétué, cette technique vocale et son répertoire ne cesseront d'évoluer, au regard de chaque génération. C'est ce qui fait toute la richesse de cette tradition orale, à travers le prisme de la diversité, du mouvement et du changement. Une tradition n'est vivante que si elle est vivace et ne peut pas être réduite à une fixation de sa pratique dans le temps, sous peine de disparition.

En parallèle aux enseignements, la retransmission radio ou télévisée, les supports enregistrés, comme le disque et l'environnement «nomade » des baladeurs Mp3 et des stockages téléphoniques de la musique participent largement à la diffusion du höömij. L'apprentissage par l'imitation et l'imprégnation opère aussi avec ces outils, en ville comme à la campagne, à l'ère du numérique. Les concerts à l'étranger et la production de disques de plus en plus importante, la sortie de clips vidéos diffusés par les chaînes de télévision et internet permettent aux höömijčid de propager leur art, leur image et de continuer à transmettre leurs pratiques autrement.

Nous avons vu que les modes de transmission du chant diphonique et les enjeux identitaires ont évolué à travers les changements sociaux que la Mongolie a connus en moins d'un siècle. D'un art pastoral, pratiqué dans des contextes spécifiques, intimement lié à la vie quotidienne, le höömij est aussi devenu un art spectaculaire à partir du début des années 1950. La Mongolie communiste (1924-1990), puis l'entrée dans le capitalisme en 1990, ont apporté de nombreux éléments caractéristiques de ce que nous connaissons aujourd'hui de cette tradition. Ce que nous entendons au xxl ${ }^{\mathrm{e}}$ siècle doit être perçu dans une dimension hybride ${ }^{33}$ constituée à la fois d'un apport du passé et des transformations vécues et intégrées au fil de l'histoire. Après les décennies du régime totalitaire, les Mongols sont à la recherche d'une certaine "authenticité mongole » à travers des traditions comme le höömij. Pourtant, il y a une trentaine d'années seulement, le chant diphonique était localisé essentiellement dans l'ouest du pays et ne concernait qu'une poignée d'individus.

48 Avec une volonté politique l'enseignement du höömij est entré à l'université. Depuis l'ouverture du pays en 1990, le concept de "patrimoine » (öv, litt. "héritage ») a cheminé dans le pensée politique mongole avec les modèles asiatiques japonais et coréen et les projets de sauvegarde des patrimoines culturels matériels et immatériels de l'Unesco. Ainsi, la question de transmettre est entrée dans un schéma plus complexe d'organisation sociale, où la politique met l'enseignement du höömij en avant, dans le projet de préserver et perpétuer un héritage, contribuant au patrimoine national. Le processus de transmission est au cœur du projet de patrimonialisation des cultures 
immatérielles (Unesco 2003, article 14). Sans hérédité, plus de patrimoine. C'est donc la fin de la présence du passé dans le présent. Nous avons vu que l'institutionnalisation de la pratique du chant diphonique et la distinction des compétences à travers la délivrance d'un diplôme professionnalisant restait un paradoxe face à la situation des détenteurs de la tradition à la campagne. Le projet politique de patrimonialisation pose donc le problème d'une reconnaissance claire des acteurs du chant diphonique sur le plan administratif. La Mongolie a visiblement des difficultés à concevoir le processus de patrimonialisation tel qu'il est décrit dans la Convention 2003 de l'Unesco, qui prône l'idée de conserver la tradition dans son mouvement dans le temps (Khaznadar 2009, p. 108). Le monde politique semble pour l'instant n'en voir dans son utilisation que l'attrait du bénéfice de ces avantages économiques (tourisme, industrie culturelle, rayonnement international). Ces exemples montrent bien le problème de la distance entre les détenteurs de la tradition et les preneurs de décisions, qui devraient considérer davantage la dimension anthropologique et les conséquences à venir de leur travail (ibid., p. 105). Mais en dehors de la place croissante qu'impose le modèle académique dans l'enseignement en milieu rural, d'organiser un grand concert à la capitale pour diffuser le höömij au niveau national, ou distribuer des médailles pour la reconnaissance des höömijčid les plus méritants, il reste à observer maintenant quelles seront les répercussions de cette inscription au Patrimoine Culturel Immatériel de l'Humanité sur la mode musicale et les pratiques du höömij dans les années à venir.

\section{Liste des témoignages}

Bapizan, B. 17/08/09, 21/09/10, dans sa maison, Cengel.

Cerendavaa, D. 14/08/04, 15/09/05, 13/08/09, sous sa yourte, Čandman'.

Cogtgerel, C. 7/10/10, dans son appartement, Ulaanbaatar.

Daržaa, D. 7/08/09, 3/09/10, sous sa yourte, Čandman'.

Enhbalsan, T.-Ö. 21/04/07, sous sa yourte, ville de Hovd.

Erdenbüren, T.-Ö. 10/09/10, sous sa yourte, Manhan.

Ganbold, T. 28/09/04, 18/08/10, salle du théâtre de l'ensemble Tümen Eh, et dans sa salle de classe, Ulaanbaatar.

Luvsanšarav, D. 4/09/11, dans son appartement, Ulaanbaatar.

Margad, G. 15/08/04, sous sa yourte, Čandman'.

Odsüren, B. année 2006-2007, 29/07/09, 6/10/10, classe de l'Université d'Art et de Culture, et dans son appartement, Ulaanbaatar.

Šagž, M. 14/08/04, dans la steppe, Čandman'.

Sengedorž, N. 10/08/04, 5/04/07, 20/08/09, théâtre de Hovd.

Tojvgoo, E. 29/08/09, 5/10/10, dans sa maison, à Ulaangom et Ulaanbaatar. 


\section{BIBLIOGRAPHIE}

Pour la bibliographie et la discographie, j'ai retranscrit les titres tels qu'ils étaient présentés par leurs auteurs. Par conséquent, la translittération des termes mongols diffère parfois de celle suivie jusqu'ici.

Aubin, F.

1967 Une expérience de collectivisation en économie nomade : la coopérative de production rurale en République Populaire de Mongolie, L'Homme et la société 4, pp. 141-148.

1993 Renouveau gengiskhanide et nationalisme dans la Mongolie postcommuniste, Cahiers d'Etudes sur la Méditerranée Orientale et le monde Turco-Iranien 16, pp. 136-204.

Baadra, Ž.

1981 Xöömej i urtyn duu : specificeskie Javlenija Mongol'skoj tradicionnoj klassiceskoj muziky, Professional'naja Muzyka Ustoj Tradicij Narodov Bliznevo Vostoka i Sovremennost (Tachkent, Izdatel'stvo literatury i iskusstva im. Gafura galiama), pp. 116-119.

1986 L'art Xöömij, Les nouvelles de Mongolie 9 (Ulaanbaatar, Mongol Press), pp. 18-19. 1998 Mongol ardyn högžim [La musique populaire mongole] (Ulaanbaatar, T\&U printing company), 168 p. Cet ouvrage posthume édité en 1998 regroupe des articles de Badraa de 1963, 1966, 1968 et 1982.

Bawden, C. R.

[1968] 1989 The Modern History of Mongolia (Londres, Kegan Paul International), 460 p.

Bulag, U. E.

1998 Nationality and Hybridity in Mongolia (Oxford, Oxford University Press), 302 p.

Ceden-Iš, A.

2009 Hög/Žim, I, Šuumž, sudlal, dursamž [La musique, vol. I, critiques, études, mémoires], et Hög\#Žil, II, Tü̈hijn tovčoon, tajlan, jarilclaga, helelcü̈lleg, helsen üg [Le développement, vol. II, chronologies, rapports, entretiens, débats, discours, 2 vol.] (Ulaanbaatar, Jikom Press hevlelijn gazar), 258 p. et 208 p.

Collectif

2003 Convention pour la sauvegarde du patrimoine culturel immatériel (Paris, Unesco), 14 p.

2011 Hün am, oron suucny 2010 ony ulsyn toollogo [Recensement national de la population et de l'habitat en 2010] (Ulaanbaatar, Comité national des statistiques de Mongolie), 274 p.

Curtet, J.

2006 Le xöömij : proposition de classification du chant diphonique mongol, chez les chanteurs professionnels, mémoire de Master, sous la dir. de H. Lacombe et le tutorat de Trân Quang Hai, 2 vol. (Rennes, Université Rennes 2 Haute-Bretagne), 214 p. + 86 p. d'annexes et 3 CD.

(à paraître) Évolution d'une tradition pastorale vers une pratique spectaculaire : le cas du chant diphonique höömij en Mongolie, Actes des journées d'études Formes spectaculaires traditionnelles et processus de patrimonialisation (25-26 mars 2010,Université Rennes 2-Haute-Bretagne), 24 p.

Curtet, J. \& Nomindar', ̌́.

(à paraître) Le PCI de Mongolie sur les listes de l'Unesco : traditions musicales et enjeux de sauvegarde, Actes des Rencontres internationales du patrimoine culturel immatériel en Bretagne (14-15 décembre 2012, Brest, Dastum/Bretagne Culture Diversité), 6 p.

Desjacques, A.

1988 Une considération phonétique sur quelques techniques vocales diphoniques mongoles, Bulletin du Centre d'Études de Musique Orientale 31, pp. 46-54. 
1992 Chants de l'Altaï mongol, thèse de doctorat sous la dir. de M. Kelkel et J. Legrand (Paris, Université Paris IV-Sorbonne), 389 p. avec une cassette audio.

2002 (avril) Fondre l'épopée : l'actualisation du répertoire épique de Mongolie Occidentale, Analyse Musicale 42, pp. 10-19.

2009 Rhapsodie en sol mongol (Lille, Le Riffle), 147 p.

Enebiš, Ž.

2001 Khumii Art is the Masterpiece of the Central Asia, in Dialogue among Civilizations : Interaction between Nomadic and Other Cultures of Central Asia (Ulaanbaatar, International Institute for the Study of Nomadic Civilizations UNESCO), pp. 160-162.

2002 The investigation of the origin of the wooden tsuur [pipe] and höomii, using ancient sources, Mongolica. An International Annual of Mongol Studies 12(33) (Ulaanbaatar, I.A.M.S.), pp. 406-409.

Doržderem, L.

2009 Dictionnaire mongol-français, mongol-franc tol', quatrième édition, revue, corrigée et augmentée (Ulaanbaatar, Université Nationale de Mongolie, École des Relations Internationales), 650 p.

Herlen, L.

2010 Mongol höömejn utga holbogdol [L'importance et le sens du höömij mongol] (Ulaanbaatar, Mongol ulsyn ih surguul'), $131 \mathrm{p}$.

Kaplonski, C.

2004 Truth, History and Politics in Mongolia. The Memory of Heroes (Londres, New-York, Routledge Curzon), $233 \mathrm{p}$.

Khaznadar, C.

2009 Les dangers qui guettent la convention de 2003, Internationale de l'Imaginaire. Le patrimoine culturel immatériel à la lumière de l'Extrême-Orient, 24 (Arles, Maison des Cultures du Monde/Actes Sud), pp. 101-109.

Legrain, L.

2007 Que sont les perceptions esthétiques en contexte mongol ?Premières balises, in Actes de la Journée d'étude "Perceptions esthétiques en contexte mongol », $3^{\mathrm{e}}$ congrès du Réseau Asie. (en ligne http://www.reseau-asie.com/colloque/thematiques-et-ateliers/journee-d-etude-perceptionsesthetiques-en-contexte-mongol/, 10 p. Consulté en novembre 2011)

2009 Le tunnel sous l'histoire. La musique et le poids moral du passé dans la Mongolie socialiste et post-socialiste, Études mongoles et sibériennes, centrasiatiques et tibétaines 40 (mis en ligne le 28 décembre 2009, http://www.emscat.revues.org/index1498.html, 19 p. Consulté en janvier 2010) 2010 Transmettre l'amour du chant ? Cris, éloquence et complaintes dans une famille ordinaire de Mongolie rurale, Terrain, Transmettre 55. (mis en ligne le 26 août 2010,http:// www.terrain.revues.org/index14055.html, pp. 54-71. Consulté en mars 2011)

2011 S'attacher à transmettre et transmettre un attachement. Les Darhad, leur répertoire et le continuum sonore en Mongolie contemporaine, thèse de doctorat en anthropologie sous la dir. de F. Lauwaert et R. Hamayon (Université Libre de Bruxelles/E.P.H.E.), 540 p.

Legrand, J. \& Legrand-Karkucinska, J.

2007 Dictionnaire français-mongol, Franc-mongol tol' (Ulaanbaatar, Monsudar), 796 p.

Léothaud, G.

1989 Considérations acoustiques et musicales sur le chant diphonique, Le Chant diphonique 1

(Limoges, Institut de la Voix), pp. 17-43.

Marsh, P. K.

2009 The Horse-head Fiddle and the Cosmopolitan Reimagination of Mongolia (New York, Routledge Press, Current Issues in Ethnomusicology Series), 240 p. 
Mergežih, F., Doržiraa, Z. \& Bajanžargal, S.

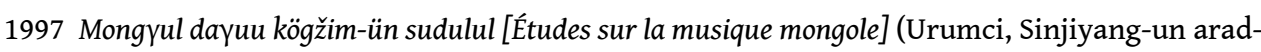
un keblel-ün qoriya), $454 \mathrm{p}$.

Pegg, C.

1991 The Revival of ethnic and Cultural Identity in West Mongolia : the Altai Uriangkhai Tsuur, the Tuvan Shuur and the Kazak Sybyzgy, Journal of the Anglo-Mongolian Society XIII(1-2), pp. 71-84. 1992 Mongolian conceptualizations of overtone singing, British Journal of Ethnomusicology 1, pp. 31-55.

2001a Mongolian Music, Dance, and Oral Narrative : Performing Diverse Identities (Seattle/Londres, University of Washington Press), 376 p. et $1 \mathrm{CD}$.

2001b Mongol music, in Stanley Sadie (éd.), The New Grove Dictionary of Music and Musicians 16 (New-York/ Londres, Stanley Sadie, seconde édition), pp. 923-934.

2001c Overtone-singing, in The New Grove Dictionary of Music and Musicians 18(New-York/Londres, Stanley Sadie, seconde édition), pp. 821-824.

2002 Mongol Music, in The Garland Encyclopedia of World Music, East Asia : China, Japan and Korea (New-York/Londres, Routledge), pp. 1003-1020.

Pouillon, J.

[1991] 2010 Tradition, in P. Bonte, M. Izard (dirs.), Dictionnaire de l'ethnologie et de l'anthropologie (Paris, Quadrige/P.U.F.), pp. 710-712.

Sandagžav, E.-O.

2010 Mongol höömej [Le höömij mongol] (Ulaanbaatar, Soyombo), 146 p.

Süzükei, V.

2009 Learning traditional music in Tuva : historical problems and contemporary solutions, in R. Sultanova (éd.), Sacred Knowledge : Schools or Revelation? Master-Apprentice System of Oral Transmission in the Music of the Turkic Speaking World (Cologne, Lambert Academic Publishing), pp. 7-16.

Tongeren van, $\mathrm{M}$.

1995 A Tuvan perspective on throat singing, in W. van Zanten et M. van Roon (éds.), Oideion : The Performing Arts of Worldwide (2). Secial issue. Ethnomusicology in the Netherlands : Present Situation and Traces of the Past (Leiden, Research School CNWS), pp. 293-312.

Trân Quang, H.

1989 Réalisation du chant diphonique, Le Chant diphonique 1 (Limoges, Institut de la Voix), pp. 15-16.

1995 Le chant diphonique : description, historique, styles, aspect acoustique et spectral, démarche originale, recherches fond amentales et appliquées, Anuario degli archivi di etnomusicologia dell' Academia Nazionale di Santa Cecilia 2 (Rome), pp. 123-150.

Zemp, H. \& Trân Quang, H.

1991 Recherches expérimentales sur le chant diphonique, Cahiers de musiques traditionnelles : Voix 4 (Genève, Georg/Ateliers d'ethnomusicologie), pp. 27-68.

\section{Discographie}

Bellenger, $\mathrm{X}$.

1986 Musique et chants de tradition populaire, Mongolie, Grem (G7511).

Bois, P. \& Gründ, F.

1989 Mongolie, musique vocale et instrumentale, MCM coll. Inédit (W 260009). 
Curtet, J.

2006 Tserendavaa et Tsogtgerel, chant diphonique de l'Altaï mongol, Routes Nomades (RN01).

2008 Tserendavaa et Tsogtgerel, Xoër Altai, Chants diphoniques de l'Altaï mongol, Buda Musique/Routes Nomades coll. Musiques du Monde (3017742).

2010 Dörvön Berkh, Four Shagai Bones, Masters of Mongolian Overtone Singing, Pan Records Ethnic Series (Pan 2100).

Desjacques, A.

1986 Mongolie : Musique et chants de l'Altaï, Orstom-Selaf (Ceto 811).

1991 Mongolie, musique traditionnelle, UNESCO Auvidis (D8207).

1995 Le chant des steppes, Talin duulal, Label Bleu (LBLC 2523).

Donner, P.

1970 Mongolia, Love Records (LREP 113).

Hamayon, R.

1973 Chants mongols et bouriates, Vogue (LMD 30 138).

Hasumi, $\mathrm{H}$.

1997 Mongolia, Living Music of the Steppes, Instrumental Music and Song from Mongolia, JVC MCM

(3001).

Jenkins, J.

[1977] 1994 Instrumental Music of Mongolia / Vocal Music of Mongolia, Tangent [TGS 126 et 127], (Topics TSCD909).

Johnston, C.

1996 Jargalant Altai. Xöömii and other vocal and instrumental music from Mongolia, Pan Records Ethnic Series (Pan 2050).

Nakagawa, Sh.

1979 Musical Voices of Asia : Mongolia, Victor Records (SJL-211).

Ormiston, $\mathrm{M}$.

2002 Chandman' Song, Amina Records (TS001).

Vargyas, L.

[1967] 1990 Mongol Nepzene, Hungaroton et Unesco [LPX 18013-14] (HCO 18013-14).

\section{Filmographie}

Badraa, Ž., Hišigt D.

1983 Mongol höömej, premier documentaire scientifique sur le höömij mongol.

Castell, J.-F.

2010 Maîtres de chant diphonique, documentaire sur mes recherches autour du höömij en Mongolie.

Webographie

URL : http://www.unesco.org/culture/ich/fr/RL/00396

Dossier de nomination de « L'art traditionnel du khöömei mongol » sur la liste représentative du Patrimoine Culturel Immatériel de l'Humanité à l'Unesco. Lien permanent, consulté en mars 2011. 


\section{NOTES}

1. Cette unique leçon de höömij a été enregistrée par A. Desjacques et éditée en 1991 sur le disque Mongolie, Musique Traditionnelle (D8207), plage 29.

2. Čandman' est un district (sum), unité territoriale administrative mongole correspondant à l'échelle d'une commune.

3. Selon elle, en dehors de son propre travail dont les résultats sont donnés dans cet article, il n'existe pas de recherche approfondie sur les processus de transmission du höömij touva. Je ne mentionne pas ici les recherches de Zoya Kyrgys, Theodore Levin et Frédéric Léotar sur le chant diphonique touva.

4. De nos jours, la pratique du höömij au Xinjiang aurait presque disparu parmi les Mongols résidants dans cette région. Mais elle a bel et bien existé, comme le montrent les recherches de F. Mergežih, Z. Doržiraa et S. Bajanžargal diffusées dans les années 1990. Les raisons de son déclin sont encore méconnues. En Mongolie-Intérieure, le chant diphonique a commencé à être enseigné à partir du début des années 1990 par Odsüren, puis dans les dix dernières années par d'autres enseignants, tous venus de Mongolie.

5. Les informations contenues dans cette définition du höömij sont tirées, en partie, de la définition que j'ai élaboré en janvier 2010 pour le dossier d'inscription du chant diphonique mongol sur la liste du Patrimoine Culturel Immatériel de l'Humanité de l'Unesco.

6. La durée d'exécution d'une mélodie de höömij dépasse rarement la minute. Dans l'apprentissage, on commence par exécuter une mélodie en la marquant de pauses respiratoires. Par la suite, les chanteurs confirmés la réalisent le plus souvent le temps d'un souffle.

7. Ž. Badraa suppose que harhiraa désigne une catégorie particulière de grue, en rapprochant le cris de cet oiseau migrateur de la technique vocale (1986, p. 18, cité par Desjacques 1992, p. 19). Mais A. Desjacques précise que le terme le plus courant pour cet animal est togruu ou togoruu (1992, p. 19, et Legrand \& Legrand-Karkucinska 2007, p. 366). Malgré cela, il propose de traduire harhiraa par « höömij grue » (ibid.). Pour m'éloigner de cette ambiguïté, je préfère utiliser le terme de "höömij profond", plus proche de la manière dont les höömijčid et musiciens mongols décrivent actuellement ce timbre de " grosse voix ». (buduun hooloj).

8. Ces techniques de modulation mentionnées à titre d'exemple ne sont pas exclusives, il en existe bien d'autres.

9. Le verbe dangildah, danildah ou dandildah désigne l'acte de chanter rythmiquement sur les syllabes dan et gild. Cela reviendrait à chanter sur l'onomatopée lalala en français. Dangild étant une ornementation syllabique du chant, je propose donc de traduire dangildah höömij par « höömij syllabique ».

10. Trân Quang Hai a aussi grandement participé à faire connaître le chant diphonique à l'échelle internationale, à travers ses nombreux stages de découverte et d'initiation à cette technique vocale.

11. En dehors de termes composés, tels que « chanter/faire/réaliser le höömij », il n'existe pas de terme simple en français pour traduire l'acte de höömijlöh. Pour alléger le texte et varier ces termes, je propose d'utiliser le néologisme "diphoner », simplifiant l'expression "chanter en chant diphonique ».

12. Jusqu'ici, cette information n'a jamais été vérifiée. Ž. Badraa a mentionné aussi l'idée que les Caatan pratiquaient le höömij, mais il semblerait qu'il n'ait pas approfondi la question (Badraa [1982] 1998, p. 46). A. Desjacques, qui a déjà effectué quelques terrains chez les Caatan, a dit ne jamais avoir rencontré de höömij dans la région du Hövsgöl (communication personnelle, été 2010). Pour certains musiciens mongols, dont B. Odsüren, le höömij des Caatan serait une pratique assez récente. Des jeunes le pratiqueraient occasionnellement, notamment pour les touristes.

13. Il s'agit des archives du théâtre dramatique de Hovd (1965-2002), des archives du théâtre d'Uliastaj à Zavhan, des Archives Nationales Centrales d'Ulaanbaatar dans les fonds concernant 
l'opéra national (1950-1975 et 1980-2002), et le théâtre dramatique (1943-1971). Cette recherche n'a malheureusement pas encore pu être complétée avec les archives des provinces d'Uvs et Bajan-Ölgij. Ce travail sur les écrits concernant le chant diphonique produits sous le communisme est en cours. Il faudrait étendre la recherche à des sources plus anciennes pour affirmer avec certitude l'absence d'informations écrites sur le höömij avant les années 1950. La période antérieure aux années 1950 et les décennies suivantes révèlent des programmes de concerts archivés par les théâtres, mettant en avant les premières utilisations du chant diphonique sur scène.

14. Concours qui sont devenus jusqu'à nos jours de véritables institutions à la campagne (Legrain, 2010 p. 66).

15. Pour davantage d'informations ethnomusicologiques sur la politique culturelle en Mongolie sous le régime communiste, cf. Pegg 2001a (chap. 4), Marsh 2009 (chap. 2 \& 3), Desjacques (2009) et Legrain $(2009,2011)$.

16. De l'ethnie hotgojd, Čimeddorž est né à Nömrög, dans la province de Zavhan. Copiste, acteur et chanteur à la chorale du théâtre de Hovd, il aurait appris le höömij de Cedee au théâtre. Il est le premier à diphoner à l'étranger dans un festival en 1957 (Sandagžav 2010, p. 40).

17. Selon les témoignages enregistrés les 14/08/04, 15/09/05 et 13/08/09.

18. Les premières compilations ethnographiques de musiques traditionnelles de Mongolie sorties à l'étranger sont: Mongol nepzene, 1967 ; Mongolia, 1970 ; Chants mongols et bouriates, 1973 ; Instrumental Music of Mongolia / Vocal Music of Mongolia, 1977 ; Musical Voices of Asia : Mongolia, 1979 ; Musique et chants de tradition populaire, Mongolie, 1986 ; Mongolie : musique et chants de l'Altaï, 1986 ; Mongolie, musique vocale et instrumentale, 1989. Depuis les années 1990, le nombre de disques consacrés à la Mongolie (à l'étranger comme dans le pays) augmente régulièrement.

19. L'idée de classifier les techniques diphoniques, présente chez la plupart des höömijčid, apparaît aujourd'hui comme un élément de la tradition höömij. Pourtant, ce sont les musicologues Ž. Badraa et Cerendavaa, grâce au tournage du film Mongol höömej (1983), qui auraient commencé à répandre cette idée parmi les chanteurs, pour clarifier et catégoriser la diversité de la pratique. Les sept sortes de höömij de Cerendavaa ont été aussi présentées auparavant par Pegg (1992, pp. 44-45).

20. Le terme de hargia serait une variante de harhiraa dans le dialecte halh de l'ouest.

21. Dans l'ordre chronologique des parutions: Musique et chants de tradition populaire, Mongolie, 1986 ; Jargalant Altai. Xöömii and other vocal and instrumental music from Mongolia, 1996 ; Mongolia, Living Music of the Steppes, Instrumental Music and Song from Mongolia, 1997 ; Chandman' Song, 2002 ; Tserendavaa et Tsogtgerel, chant diphonique de l'Altaï mongol, 2006 ; Tserendavaa et Tsogtgerel, Xoër Altai, Chants diphoniques de l'Altaï Mongol, 2008 ; Dörvön Berkh, Four Shagai Bones, Masters of Mongolian Overtone Singing, 2010.

22. Pour leur caractère scientifique, citons notamment: Mongol höömej de Ž. Badraa, 1983 ; Les steppes insolites de Mongolie de H. Draper, 1994 ; Maîtres de chant diphonique de J.-F. Castell, 2010.

23. Selon les témoignages des deux frères enregistrés respectivement les 21/04/07 et 10/09/10.

24. Cette rivalité aurait commencé après que les premiers höömijčid touva et mongols soient entrés dans le système des tournées et des productions de disques. Ce discours est présent chez les höömijčid professionnels ou les enseignants, mais se retrouve peu à la campagne. L'intérêt de cette paternité semblerait être seulement la préoccupation des musiciens de scène en concurrence.

25. Pegg rapporte qu'avant 1910, la population kazakhe ne dépassait pas l'échelle d'un village dans la province de Bajan-Ölgij (1991, p. 72). Depuis cette période le nombre n'a fait qu'augmenter, selon le Comité national des statistiques de Mongolie (2011, pp. 56-57)

26. Selon son témoignage enregistré le 21/09/10.

27. Ž. Badraa a joué un grand rôle dans l'étude des musiques traditionnelles de Mongolie à la période communiste. Il ne partageait pas toujours les idées du Parti et tentait parfois de 
conserver un certain regard objectif dans ses travaux (Marsh 2009, pp.97-99). Malgré cela, il a contribué a mettre en avant la pratique du höömij des Halh, en encourageant les höömijčid de ce groupe plus que les autres, en désignant Čandman' (district peuplé de Halh) comme un berceau de la tradition à travers son film Mongol höömej et en instaurant progressivement des séries de cours de höömij à Ulaanbaatar, avec des enseignants halh uniquement, comme Cerendavaa, Ganbold, puis Odsüren qu'il introduisit à l'Université. Cette analyse apparaissait limpide à travers le discours de la plupart de mes informateurs entendus sur le terrain depuis 2004.

28. À Touva, l'institutionnalisation de l'enseignement du höömij a aussi commencé en 1992 et 1993 (Süzükei 2009, p. 9).

29. J'ai eu l'opportunité de passer une année en classe de chant diphonique dans cette université, grâce à une bourse EGIDE du Ministère des Affaires Étrangères, en 2006-2007. Cela m'a permis d'observer de près l'enseignement d'Odsüren.

30. Selon Odsüren, il est naturel d'imiter les sons qui nous entourent.

31. Les enregistrements d'archive conservés à la Radio Nationale de Mongolie datant des années 1950 et 1960 montrent que les chanteurs de höömij n'utilisaient à cette époque qu'une seule technique diphonique pour une mélodie donnée, le höömij sifflé (isgeree höömij).

32. On retrouve aussi ces fondements (les liens multiples entre le chant et la nature, la nature comme source d'inspiration) au centre de l'éducation et de l'attention portée sur la voix dans la Mongolie rurale (Legrain 2010, 2011).

33. Cette notion d'hybridité de la musique se retrouve dans les travaux de Pegg 2001a, Marsh 2009 et Legrain 2009.

\section{RÉSUMÉS}

Transmis par l'oralité de génération en génération dans les provinces de l'Altaï, l'enseignement du chant diphonique mongol (höömij) est aussi entré dans les milieux urbains et la sphère institutionnelle de l'université dans les années 1990. Face aux changements sociaux rapides que la Mongolie a connus en moins d'un siècle, d'importantes transformations ont été apportées au chant diphonique. À travers trois générations, les pratiques de transmission du höömij ont évolué et des enjeux identitaires se sont progressivement installés entre les chanteurs de höömij, mais aussi dans le monde politique. La transmission du chant diphonique n'est plus la même lorsque l'on passe du local au global ni quand le höömij fait l'objet d'une inscription sur la liste représentative du Patrimoine Culturel Immatériel de l'Unesco.

Transmitted by orality from generation to generation in the Altai provinces, the teaching of Mongolian overtone singing (höomij) also entered in the urban areas and the institutional sphere of the university in the 1990s. Faced with rapid social changes that Mongolia has experienced in less than a century, major changes occurred in overtone singing. Through three generations, the practices of höomij transmission have evolved and identity issues have been gradually installed between the höomij singers, but also in the politic. The overtone singing's transmission is not the same when we pass from local to global and when höomij is inscribed on the representative list of Intangible Cultural Heritage of Unesco. 


\section{INDEX}

Index géographique : Mongolie

Keywords : ethnomusicology, music, overtone singing, höömij, transmission, teaching, heritage nomsmotscles Halh, Bayad, Touva, Dörvöd

Mots-clés : ethnomusicologie, musique, chant diphonique, höömij, transmission, enseignement, patrimonialisation, Zahčin, Altaj Urianhaj, Hotgojd

\section{AUTEUR}

\section{JOHANNI CURTET}

Johanni Curtet est doctorant en musicologie à l'Université de Rennes 2-Haute Bretagne. Ses recherches portent sur les styles et techniques du chant diphonique mongol (höömij), son histoire, sa transmission ainsi que la patrimonialisation de cette tradition. De 2004 à 2010, plusieurs bourses (Egide, Fondation Internationale Nadia et Lili Boulanger, Aires Culturelles, Collège Doctoral International de l'Université Européenne de Bretagne, American Center for Mongolian Studies, Aide au terrain de la Société Française d'Ethnomusicologie) lui ont permis d'effectuer ses recherches. En 2010, à la demande de la commission nationale de la Mongolie pour l'Unesco, il a participé à l'inscription du chant diphonique mongol sur la liste représentative du Patrimoine Culturel Immatériel de l'Humanité.

curtetjohanni@yahoo.fr 